\title{
Preference programming approach for solving intuitionistic fuzzy AHP
}

\author{
Bapi Dutta , Debashree Guha \\ Department of Mathematics, Indian Institute of Technology, Patna, \\ Bihar 800013, India \\ E-mail:bdutta@iitp.ac.in,debashree@iitp.ac.in
}

Received 16 April 2015

Accepted 7 August 2015

\begin{abstract}
The aim of this paper is to present a novel approach for deriving weights of the decision criteria or alternatives in multi-attribute decision making (MADM) under intuitionistic fuzzy (IF) environment. In order to tackle the uncertainty and imprecision of the practical situations, decision makers' pair-wise comparison judgments are represented by intuitionistic fuzzy numbers (IFNs). The assessment of the priorities from these IF pair-wise comparison judgments is formulated as an IF decision making problem where goals are described in intuitionistic fuzzy sense. Then by resolving hesitancy via a parameter, IF goals are transformed into fuzzy goals. Finally, aggregation of fuzzy goals and application of the max - min principle lead us to a nonlinear optimization problem whose solution gives the desired crisp priorities. Unlike the other prioritization methods, the proposed approach generates crisp priorities from IF pair-wise comparison matrix. Thus, the proposed approach eliminates the additional requirement of arithmetic operations of IFNs for aggregation and also avoids the ranking process of IFNs to compare the final IF priorities, which may produce ambiguous results. Finally, examples are given to illustrate the proposed process with an application to MADM problem.
\end{abstract}

Keywords: AHP, Intuitionistic fuzzy numbers, Decision making,

\section{Introduction}

The rapid changes in the perceived world and the growing complexity of socio-economic environment are making the task of taking suitable decisions in the presence of multiple conflicting attributes more complicated day by day. Various approaches have been developed to solve such complex multiattribute decision making (MADM) problems which frequently arise in management science and operations research. Among them, Analytic Hierarchy Process (AHP), originally introduced by Saaty 1,2 , is one of the most popular and widely used MADM techniques. The AHP aids the decision makers to solve complex decision making problems by struc- turing them into a simple and comprehensible multilevel hierarchical structure of goals, attributes, subattributes and alternatives. After creating hierarchical structure, the components of each level are compared in pair-wise fashion by using Saaty's '1-9' point scale, where ' 1 ' denotes that two components are equally important and ' 9 ' represents that one component is absolutely important than the other. Subsequently, pair-wise comparison matrix is constructed from which local priorities are derived. Finally, by aggregating all the local priorities, global priorities of the alternatives are derived for ranking the alternatives.

In traditional AHP, decision makers provide their preferences for pair-wise comparison judgments in 
Table 1: The different approaches of deriving priorities from fuzzy pair-wise comparison matrix

\begin{tabular}{|c|c|}
\hline Proposed by & Prioritization methods \\
\hline Van Laarhoven and Pedrycz (1983) & $\begin{array}{l}\text { Uses logarithmic least square } \\
\text { method to derive fuzzy priorities } \\
\text { and performance scores }\end{array}$ \\
\hline Buckley (1985) & $\begin{array}{l}\text { Uses geometric mean approach } \\
\text { to derive fuzzy priorities and } \\
\text { performance scores }\end{array}$ \\
\hline Bonder et al. (1989) & $\begin{array}{l}\text { Modifies Van Laarhoven and } \\
\text { Pedrycz's approach via robust } \\
\text { normalization procedure of local } \\
\text { priorities }\end{array}$ \\
\hline Chang (1996) & $\begin{array}{l}\text { Uses synthetic extent analysis } \\
\text { with possibility degree compar- } \\
\text { ison method of triangular fuzzy } \\
\text { numbers to derive crisp priori- } \\
\text { ties and scores }\end{array}$ \\
\hline Csutora and Buckley (2001) & $\begin{array}{l}\text { Extends } \lambda_{\max } \text { method proposed } \\
\text { by satty's in AHP under fuzzy } \\
\text { environment via } \alpha \text {-cut of fuzzy } \\
\text { numbers }\end{array}$ \\
\hline Mikhailov (2003) & $\begin{array}{l}\text { Uses fuzzy linear preference } \\
\text { programming method to derive } \\
\text { crisp priorities based on } \alpha \text {-cut of } \\
\text { fuzzy numbers }\end{array}$ \\
\hline Mikhailov (2004) & $\begin{array}{l}\text { Uses fuzzy non-linear prefer- } \\
\text { ence programming method to } \\
\text { derive crisp priorities }\end{array}$ \\
\hline Wang et al. (2006) & $\begin{array}{l}\text { Modifies Bonder et al.'s ap- } \\
\text { proach by taking more robust } \\
\text { normalization method of local } \\
\text { priorities }\end{array}$ \\
\hline
\end{tabular}

precise form. However, in many situations due to lack or abundance of information, subjective estimation or vagueness and incomplete knowledge about the complex system decision makers' preferences may not be assessed with both precision and certainty. A natural way to cope with such vagueness and uncertainty present in human judgments is expressing them by using fuzzy numbers. In this regard, crisp prioritization problems transform into fuzzy prioritization problems. Several methods have been proposed in the literature to solve the prioritiza- tion problems under fuzzy environment. Laarhoven and Pedrycz ${ }^{3}$ are the first researchers to use triangular fuzzy numbers for describing vague ratios of pair-wise comparisons and proposed a logarithmic least square method to derive fuzzy priorities and fuzzy global priorities for ranking the alternatives. Wagenknecht and Hartmann ${ }^{4}$ introduced the geometric mean approach to derive fuzzy priorities from complete fuzzy pair-wise comparison matrix. Buckley ${ }^{5}$ extended the original AHP with trapezoidal fuzzy numbers and obtained fuzzy priorities 
via geometric mean approach. Chang ${ }^{6}$ introduced extent analysis method to derive crisp priorities from the fuzzy pair-wise comparison matrix. Apart from these above mentioned well known approaches of deriving priority vector from fuzzy pair-wise comparison matrix, many methods have been proposed in literature $7,8,9,10,11,12,13$. Table 1 describes different prioritization methods proposed in literature to derive priorities from fuzzy pair-wise comparison matrix.

In the aforementioned literature of fuzzy AHP, mainly two kinds of fuzzy numbers, namely, triangular and trapezoidal fuzzy numbers are used to model the uncertainty of pair-wise comparison ratios. The fuzzy number is characterized by membership function which captures decision maker's satisfaction for taking different values of the comparison ratios from the support of the corresponding fuzzy numbers. However, in many practical situations, only membership function is inadequate to describe the uncertainty (i.e., hesitation) related to human cognitive processes viz., thinking, reasoning, etc. Intuitionistic fuzzy set (IFS), introduced by Atanassov ${ }^{14}$, is characterized by membership and non-membership functions and, thus, can depict the uncertainty and vagueness of human's judgments in a more intuitive way than fuzzy set. Owing to this fact, the concepts of IFS in traditional AHP were introduced through pair-wise comparisons by Sadiq and Tesfamariam ${ }^{15}$. In their paper, the uncertainty in pair-wise comparison ratios was suitably modeled by triangular intuitionistic fuzzy number (TIFN). In this process the priorities are computed as TIFNs by using large numbers of addition and multiplication operations on the original decision information, and, thus, the resultant TIFNs possess large support and overlap over a large range. Hence, the initial preference of the decision makers may not be preserved, which produces inconsistent results. Moreover, the method mentioned above required an additional ranking procedure to compare the final intuitionistic fuzzy priorities (or weights). Different ranking procedures, however often provide different ranking results.

To overcome these demerits of the existing method, inspired by the fuzzy prioritization method of Mikhailov and Tsvetinov ${ }^{11}$ and its improvement by Rezaei et al. ${ }^{16}$, we propose an intuitionistic fuzzy preference programming (IFPP) approach to derive crisp priority vector from intuitionistic fuzzy pair-wise comparison matrix. The proposed IFPP approach does not require large numbers of arithmetic operations on IFNs. The IFPP approach is applied to transform the preference programming into an intuitionistic fuzzy optimization problem which can derive crisp priority from given intuitionistic fuzzy judgments. Thus, the proposed approach can eliminate additional requirement of ranking procedure which sometimes produce ambiguous results.

On the other hand, in modeling a real-life decision making situation it is important to know the confidence level of the decision maker regarding his/her opinion. For instance, decision maker might have 'high' level of confidence on the comparison ratio of the factor 1 over factor 2 , while he/she might be 'moderately' confident on the comparison ratio of the factor 1 over factor 3 . It is evident that decision maker has different level of confidence associated with comparison of different factors. Therefore, considering the level of confidence in decision maker's judgment is an important aspect during the process of constructing pair-wise comparison matrix. By conducting a concise review of the existing fuzzy approaches, it is observed that the problem of weight computation, considering the confidence level of decision makers, in the pair-wise comparison judgments, has not yet been attended so far. This is the aspect that has motivated us to model decision maker's judgments by generalized triangular intuitionistic fuzzy numbers (GTIFNs) and, thus, it would allow us to capture the decision maker's confidence and non-confidence levels effectively.

To do this, rest of the paper is organized as follows. In section 2, we present a brief primer of IFNs and their arithmetic operations. Section 3 proposes intuitionistic fuzzy preference programming (IFPP) methodology for deriving priority vector from pairwise comparison matrix whose entries are GTIFNs. Numerical examples are presented to illustrate the proposed approach in section 4 . The paper concludes in section 5 . 


\section{Basic concepts of intuitionistic fuzzy AHP}

\subsection{Intuitionistic fuzzy numbers}

Definition 1. Let $X \subseteq \mathbb{R}$ be a non-empty set, where $\mathbb{R}$ is the set of real number. An Atanassov's intuitionistic fuzzy set $\widetilde{A}$ over $X$ is defined as ${ }^{14}$

$$
\widetilde{A}=\left\{\left(x, \mu_{\widetilde{A}}(x), v_{\widetilde{A}}(x)\right) \mid x \in X\right\}
$$

where $\mu_{\tilde{A}}, v_{\tilde{A}}: X \rightarrow[0,1]$, define the membership and non-membership functions, respectively. For every $x \in X: \mu_{\widetilde{A}}(x)+v_{\widetilde{A}}(x) \leqslant 1$. Moreover, $\mu_{\widetilde{A}}(x)$ and $v_{\widetilde{A}}(x)$ indicate the degree of membership and non-membership of an element $x \in X$ to the intuitionistic fuzzy set $\widetilde{A}$, respectively.

It is easily observed that when $\mu_{\widetilde{A}}(x)+v_{\widetilde{A}}(x)=1$ for all $x \in X$, then intuitionistic fuzzy set $\widetilde{A}$ degenerates to a fuzzy set $\widetilde{A}$, which can be represented as follows:

$$
\widetilde{A}=\left\{\left(x, \mu_{\widetilde{A}}(x)\right) \mid x \in X\right\}
$$

The quantity $\pi_{\widetilde{A}}(x)=1-\mu_{\widetilde{A}}(x)-v_{\widetilde{A}}(x)$ is called the degree of non-determinancy or hesitancy of an element $x \in X$ to the intuitionistic fuzzy set $\widetilde{A}$.

Definition 2. A generalized triangular intuitionistic fuzzy number (GTIFN) $\widetilde{A}=\left\langle(l, m, u) ; \gamma_{\widetilde{A}}, \delta_{\widetilde{A}}\right\rangle$ is a special IF set whose membership $\left(\mu_{\tilde{A}}\right)$ and nonmembership $\left(v_{\widetilde{A}}\right)$ functions can be defined as ${ }^{17,18,19}$

$$
\mu_{\widetilde{A}}(x)= \begin{cases}\frac{x-l}{m-l} \gamma_{\tilde{A}} & \text { if } l \leqslant x<m, \\ \gamma_{\tilde{A}} & \text { if } x=m, \\ \frac{u-x}{u-m} \gamma_{\tilde{A}} & \text { if } m<x \leqslant u \\ 0 & \text { otherwise }\end{cases}
$$

and

$$
v_{\widetilde{A}}(x)= \begin{cases}\frac{m-x+\delta_{\tilde{A}}(x-l)}{m-l} & \text { if } l \leqslant x<m \\ \frac{\delta_{\widetilde{A}}}{x-m+\delta_{\tilde{A}}(u-x)} & \text { if } x=m \\ 1 & \text { if } m<x \leqslant u\end{cases}
$$

respectively. The values $\gamma_{\widetilde{A}}$ and $\delta_{\widetilde{A}}$ denote the maximum value of membership degree and minimum value of non-membership degree, respectively, and they satisfy the conditions: $\gamma_{\tilde{A}} \geqslant 0, \delta_{\widetilde{A}} \geqslant 0, \mu_{\widetilde{A}}+$ $v_{\widetilde{A}} \leqslant 1$.

If we set $\delta_{\widetilde{A}}=0$, then GTIFN $\widetilde{A}$ degenerates to $\widetilde{A}=\left\langle(l, m, u) ; \gamma_{\tilde{A}}, 0\right\rangle$, which is nothing but just generalized triangular fuzzy numbers with height $\gamma_{\tilde{A}}$. Moreover, when $\gamma_{\tilde{A}}=1$ with $\delta_{\widetilde{A}}=0$, GTIFN $\widetilde{A}$ reduces to a normal triangular fuzzy number (TFN) $(l, m, u)$.

A positive real number $l$ can also be represented by a GTIFN, $\widetilde{A}=\langle(l, l, l) ; 1,0\rangle$. The maximum membership degree $\gamma_{\hat{A}}=1$ ensures that decision maker has no hesitation on his/her subjective judgments.

It is also noted that GTIFN uses two additional parameters $\gamma_{\widetilde{A}}$ and $\delta_{\widetilde{A}}$ to reflect decision maker's confidence and non-confidence in a more comprehend way. Thus, in context of decision making, GTIFN would be more capable of modeling humans perception, thinking, reasoning etc. than TFN representation. In many practical situations, it is also observed that the decision maker may not be able to get proper decision results based on uncertain/imprecise information quantified by TFN. In these circumstances, remodeling of the available decision information is required and GTIFNs provide a more appropriate tool to model such uncertain observation.

As our intention is to express the uncertain pair-wise comparison ratio more suitably by using GTIFN, we will restrict ourselves to positive GTIFNs. By positive GTIFNs we mean that support of GTIFN is a subset of positive real numbers, $\mathbb{R}^{+}$. Formally, it can be defined as follows:

Definition 3. A GTIFN $\widetilde{A}=\left\langle(l, m, u) ; \gamma_{\tilde{A}}, \delta_{\widetilde{A}}\right\rangle$ is said to be positive if $l>0$.

The arithmetic operations between two positive GTIFNs $\widetilde{A}_{1}=\left\langle\left(l_{1}, m_{1}, u_{1}\right) ; \gamma_{\widetilde{A}_{1}}, \delta_{\widetilde{A}_{1}}\right\rangle$ and $\widetilde{A}_{2}=$ $\left\langle\left(l_{2}, m_{2}, u_{2}\right) ; \gamma_{\widetilde{A}_{2}}, \delta_{\widetilde{A}_{2}}\right\rangle$ can be facilitated by utilizing following laws ${ }^{17}$ :

- Addition $\widetilde{A}_{1} \oplus \widetilde{A}_{2}=\left\langle\left(l_{1}+l_{2}, m_{1}+m_{2}, u_{1}+u_{2}\right)\right.$; $\left.\left.\left.\gamma_{\widetilde{A}_{1}} \wedge \gamma_{\widetilde{A}_{2}}\right), \delta_{\widetilde{A}_{1}} \vee \delta_{\widetilde{A}_{2}}\right)\right\rangle$

- Multiplication $\widetilde{A}_{1} \otimes \widetilde{A}_{2}=\left\langle\left(l_{1} l_{2}, m_{1} m_{2}, u_{1} u_{2}\right) ; \gamma_{\widetilde{A}_{1}} \wedge\right.$ $\left.\left.\gamma_{\widetilde{A}_{2}}, \delta_{\widetilde{A}_{1}} \vee \delta_{\widetilde{A}_{2}}\right)\right\rangle$

- Division $\widetilde{A}_{1} / \widetilde{A}_{2}=\left\langle\left(l_{1} / u_{2}, m_{1} / m_{2}, u_{1} / l_{2}\right) ; \gamma_{\tilde{A}_{1}} \wedge\right.$ $\left.\left.\gamma_{\widetilde{A}_{2}}, \delta_{\widetilde{A}_{1}} \vee \delta_{\widetilde{A}_{2}}\right)\right\rangle$ 
where $\wedge$ denotes the 'min' operator and $\vee$ represents the 'max' operator. One may also verify that the above operational laws are the natural extension of arithmetic operation laws of generalized fuzzy numbers ${ }^{20}$.

Based on division law, the reciprocal of a GTIFN can be defined as follows ${ }^{17}$ :

Definition 4. Let $\widetilde{A}=\left\langle(l, m, u) ; \gamma_{\widetilde{A}}, \delta_{\widetilde{A}}\right\rangle$ be a positive GTIFN. Then

$$
A^{-1}=\left\langle(1 / u, 1 / m, 1 / l) ; \gamma_{\tilde{A}}, \delta_{\widetilde{A}}\right\rangle
$$

\section{Intuitionistic fuzzzy preference programming (IFPP)}

In this section, we shall first describe how we interpret GTIFN for pair-wise comparison scale.

\subsection{Presentation of generalized intuitionistic fuzzy numbers for pair-wise comparison scale}

In the context of intuitionistic fuzzy AHP, after structuring the hierarchy of the complex decision problems, the first task is to compare the elements in the same hierarchy level and assign the relative importance to each pair of elements. When decision maker faces the complex and uncertain problem, he/she prefers to express the comparison judgments as uncertain ratios, such as, 'about two times more important', 'between two and four times less important', etc. The confidence of the decision makers is also intrinsically connected to their judgments. Such kinds of uncertain ratios can be more effectively modeled by GTIFN as it provides more flexibility to decision maker by incorporating hesitation in judgments.

By using GTIFNs, suppose decision maker constructs the pair-wise comparison matrix, $\widetilde{A}=$ $\left(\widetilde{a}_{i j}\right)_{n \times n}$, where $\widetilde{a}_{i j}$ denotes the uncertain relative importance of the factor $i$ over the factor $j$. For instance, if $\widetilde{a}_{i j}=\left\langle\left(l_{i j}, m_{i j}, u_{i j}\right) ; \gamma_{\widetilde{a}_{i j}}, \delta_{\widetilde{a}_{i j}}\right\rangle$ is used to express an uncertain pair-wise comparisons ratio of factor $i$ over factor $j$, then the triplet $\left(l_{i j}, m_{i j}, u_{i j}\right)$ represents minimum, most likely and maximum values of the ratio, respectively with decision maker's different satisfaction, dissatisfaction and hesitation degrees. For most likely value $m_{i j}$, decision maker's satisfaction degree becomes maximum, i.e., $\gamma_{a_{i j}}$ and dissatisfaction degree becomes minimum, i.e., $\delta_{\widetilde{a}_{i j}}$ with minimum hesitation degree, i.e., $\pi_{\widetilde{a}_{i j}}\left(m_{i j}\right)=$ $1-\gamma_{a_{i j}}-\delta_{\widetilde{a}_{i j}}$. It is worth to mention that the degree of confidence and degree of non-confidence are intrinsically connected to decision maker's opinion. Therefore, by associating decision maker's confidence and non-confidence levels into his/her opinion, the values of $\gamma_{\widehat{a}_{i j}}$ and $\delta_{\widetilde{a}_{i j}}$ are realized. Again, at minimum and maximum values of ratios, decision maker's satisfaction becomes zero, i.e., he/she is completely dissatisfied. Similarly, the importance of the factor $j$ over $i$ can be represented via reciprocal of the GTIFN $\widetilde{a}_{i j}$, i.e., $\widetilde{a}_{j i}=\widetilde{a}_{i j}^{-1}=$ $\left\langle(1 / u, 1 / m, 1 / l) ; \gamma_{\hat{a}_{i j}}, \delta_{\tilde{a}_{i j}}\right\rangle$ and interpreted as earlier. It is important to note here that the satisfaction and dissatisfaction of the decision maker regarding the judgment of importance of factor $i$ over factor $j$ and again the importance of factor $j$ over factor $i$ are kept same following the reciprocal law. In this way, GTIFN can model decision maker's satisfaction, dissatisfaction and hesitation of subjective judgments on pair-wise comparisons in a more meaningful way than fuzzy numbers. With this interpretation in background, we shall now provide the methodology for deriving crisp priority vector from the pair-wise comparison matrix with GTIFNs entries.

\subsection{Problem statement}

The intuitionistic fuzzy prioritization problem with $n$ elements can be defined as deriving the unknown priorities $w=\left(w_{1}, w_{2}, \ldots, w_{n}\right)^{T}$, such that $w_{i}$ 's are positive real numbers, from the intuitionistic fuzzy pair-wise comparison matrix $\widetilde{A}=\left(\widetilde{a}_{i j}\right)_{n \times n}$ where the pair-wise comparisons of $n$ elements, given by the decision makers, are modeled by using positive GTIFNs, $\widetilde{a}_{i j}(i, j=1,2, \ldots, n)$. Thus, a generalized intuitionistic fuzzy pair-wise comparison matrix can 
be expressed as

$$
\widetilde{A}=\left(\begin{array}{ccc}
\widetilde{a}_{11} & \widetilde{a}_{12} \cdots & \widetilde{a}_{1 n} \\
\widetilde{a}_{21} & \widetilde{a}_{22} \cdots & \widetilde{a}_{2 n} \\
\vdots & \vdots \cdots & \vdots \\
\widetilde{a}_{n 1} & \widetilde{a}_{n 2} \cdots & \widetilde{a}_{n n}
\end{array}\right)
$$

where $\quad \tilde{a}_{i j}=\left\langle\left(l_{i j}, m_{i j}, u_{i j}\right) ; \gamma_{i j}, \delta_{i j}\right\rangle \quad, \quad \tilde{a}_{j i}=$ $\left\langle\left(l_{j i}, m_{j i}, u_{j i}\right) ; \gamma_{j i}, \delta_{j i}\right\rangle=\left\langle\left(1 / u_{i j}, 1 / m_{i j}, 1 / l_{i j}\right) ; \gamma_{i j}\right.$, $\left.\delta_{i j}\right\rangle, \gamma_{i j} \geqslant 0 \delta_{i j} \geqslant 0$ with $\gamma_{i j}+\delta_{i j} \leqslant 1$ for all $i, j=1,2, \ldots, n$ and $\widetilde{a}_{i i}=\langle(1,1,1) ; 1,0\rangle$ for all $i=1,2, \ldots, n$.

Our aim is to derive a crisp priority vector $w=$ $\left(w_{1}, w_{2}, \ldots, w_{n}\right)^{T}$ in such a way that the priority ratio $w_{i} / w_{j}$ is approximately within the scopes of initial intuitionistic fuzzy judgments for all $i, j=1,2, \ldots, n$ and $i \neq j$ or equivalently,

$$
l_{i j} \lesssim w_{i} / w_{j} \lesssim u_{i j}
$$

We shall call 'ミ’ as 'less than or equal to' type inequality in intuitionistic fuzzy sense and its interpretation is provided in the following section.

\subsection{Assumptions of the IFPP}

The intuitionistic fuzzy inequality Eq. (5) can be characterized as an IFS given by

$$
G_{i j}=\left(\mu_{i j}\left(w_{i} / w_{j}\right), v_{i j}\left(w_{i} / w_{j}\right)\right)
$$

where the membership and non-membership functions are to be understood in the following sense describe below.

In order to measure decision maker's satisfaction and dissatisfaction degrees for different values of the ratio $w_{i} / w_{j}$, we introduce membership $\left(\mu_{i j}\left(w_{i} / w_{j}\right)\right)$ and non-membership $\left(v_{i j}\left(w_{i} / w_{j}\right)\right)$ functions. Each priority vector satisfies double sided intuitionistic fuzzy inequality Eq. (5) with a certain satisfaction degree which can be represented by the following linear membership function

$$
\mu_{i j}\left(w_{i} / w_{j}\right)=\left\{\begin{array}{l}
\frac{w_{i} / w_{j}-l_{i j}}{m_{i j}-l_{i j}} \gamma_{i j}, \frac{w_{i}}{w_{j}} \leqslant m_{i j} \\
\frac{u_{i j}-w_{i} / w_{j}}{u_{i j}-m_{i j}} \gamma_{i j}, \frac{w_{i}}{w_{j}} \geqslant m_{i j}
\end{array}\right.
$$

From the construction of the membership function, we observe that it is increasing in $\left(-\infty, m_{i j}\right)$ and decreasing in $\left(m_{i j}, \infty\right)$. The membership function also attains its maximum value $\mu_{i j}\left(w_{i} / w_{j}\right)=\gamma_{i j}$ at $w_{i} / w_{j}=m_{i j}$. Over the interval $\left[l_{i j}, u_{i j}\right]$, the membership function described in Eq. (7) perfectly coincides with the membership function of the GTIFN $\widetilde{a}_{i j}=\left\langle\left(l_{i j}, m_{i j}, u_{i j}\right) ; \gamma_{i j}, \delta_{i j}\right\rangle$. It can take negative values outside the interval $\left[l_{i j}, u_{i j}\right]$.

Similarly, for measuring the dissatisfaction degree for different values of the ratios $w_{i} / w_{j}$, we construct non-membership function as follows:

$$
v_{i j}\left(w_{i} / w_{j}\right)=\left\{\begin{array}{l}
\frac{m_{i j}-w_{i} / w_{j}+\delta_{i j}\left(w_{i} / w_{j}-l_{i j}\right)}{m_{i j}-l_{i j}}, \frac{w_{i}}{w_{j}} \leqslant m_{i j} \\
\frac{w_{i} / w_{j}-m_{i j}+\delta_{i j}\left(u_{i j}-w_{i} / w_{j}\right)}{u_{i j}-m_{i j}}, \frac{w_{i}}{w_{j}} \geqslant m_{i j}
\end{array}\right.
$$

Clearly, non-membership function is monotone decreasing in the interval $\left(-\infty, m_{i j}\right)$ and monotone increasing in the interval $\left(m_{i j}, \infty\right)$. It attains minimum value $v_{i j}\left(w_{i} / w_{j}\right)=\delta_{i j}$ at $w_{i} / w_{j}=m_{i j}$. We also note that the non-membership function, defined in Eq. (8), coincides with the non-membership function of the GTIFN $\widetilde{a}_{i j}=\left\langle\left(l_{i j}, m_{i j}, u_{i j}\right) ; \gamma_{i j}, \delta_{i j}\right\rangle$ in the interval $\left[l_{i j}, u_{i j}\right]$ and outside this interval $v_{i j}$ takes value greater than or equal to one.

Now, we are going to associate, a function, $\pi_{i j}\left(w_{i} / w_{j}\right)=1-\mu_{i j}\left(w_{i} / w_{j}\right)-v_{i j}\left(w_{i} / w_{j}\right)$ with each of the ratios $w_{i} / w_{j}$ corresponding to the priority vector $w$ to indicate decision maker's indeterminacy/hesitation in assigning corresponding satisfaction and dissatisfaction degrees. We also note that $\pi_{i j}\left(w_{i} / w_{j}\right) \in[0,1]$ when $w_{i} / w_{j} \in\left[l_{i j}, m_{i j}\right]$ and outside the interval it takes negative values.

Thus, with the help of the constructed membership and non-membership functions associated with each ratio $w_{i} / w_{j}$ corresponding to the crisp priority vector $w$, we can express the satisfaction and dissatisfaction of the IF inequality, namely, Eq. (5), in terms of intuitionistic fuzzy goal describing through IFS, $G_{i j}$. So, the determination of crisp priority vector $w=\left(w_{1}, w_{2}, \ldots, w_{n}\right)$, which basically aims to determine the priority vector, having the highest degree of membership and the lowest degree of nonmembership, leads to an optimization problem with intuitionistic fuzzy goals $G_{i j} ; i, j=1,2, \ldots, n, i \neq j$ under a crisp constraint on $n-1$ dimensional simplex. A $n-1$ dimensional simplex is denoted as $Q^{n-1}$ and defined as follows: 


$$
Q^{n-1}=\left\{w=\left(w_{1}, w_{2}, \ldots, w_{n}\right) \mid w_{i} \geqslant 0, \sum_{i=1}^{n} w_{i}=1\right\}
$$

\subsection{Optimization method for finding crisp priority vector}

The IFPP problem is now transformed into an optimization problem with the IF objective (Eq. (6)) and crisp constraints (Eq. (9)). The first serious attempt to use IFS in optimization problems was made by Angelov ${ }^{21}$. Later, Yager ${ }^{22}$ pointed out certain difficulties of this method and in 2012, Dubey et al. ${ }^{23}$ modified the intuitionistic fuzzy optimization model ${ }^{21}$ as suggested by Yager. In this present study, we will utilize this approach proposed by Dubey et al. ${ }^{23}$ to solve our prioritization problem describing through IF goals $G_{i j} ; i, j=1,2, \ldots, n, i \neq j$ and a crisp constraint, $Q^{n-1}$.

The solution procedure of the decision making problem with intuitionistic fuzzy goals and constraints can be described as a two-stage process which includes aggregation of goals and constraints and then defuzzification to form the corresponding crisp model. As suggested by Dubey et al. ${ }^{23}$ before the aggregation step takes place, the first aim is to resolve hesitation factors associated with each of the IF goals $G_{i j}$ and constraints.

Let $D$ denotes IF decision space which is the intersection of IF goals $G_{i j} ; i, j=1,2, \ldots, n, i \neq j$ and the crisp constraint $Q^{n-1}$ based on priority vector $w$. Now, we are going to associate a value function, corresponding to each ratio of the priority vector $w \in Q^{n-1}$, which is defined below.

Definition 5. For every priority vector $w \in Q^{n-1}$, we associate a value function corresponding to each ratio $w_{i} / w_{j}(i, j=1,2, \ldots, n, i \neq j)$ as follows:

$F_{i j}\left(w_{i} / w_{j}\right)=\mu_{i j}\left(w_{i} / w_{j}\right)+\lambda \pi_{i j}\left(w_{i} / w_{j}\right), \lambda \in[0,1]$.

By choosing appropriate value of $\lambda$, the decision maker first resolves the hesitancy associated with each of the pair-wise judgments. Moreover, the larger value of $\lambda$ indicates that the decision maker resolves more indeterminacy in favor of membership, while lower values of $\lambda$ suggests that the decision maker resolves more indeterminacy in favor of non-membership. Once the ambiguity factor disappears through $\lambda$, the IF decision space $D$ transforms into a fuzzy decision space $D_{\lambda}$ whose membership function for each ratio $w_{i} / w_{j}$ corresponding to the priority vector $w$ is described by $F_{i j}\left(w_{i} / w_{j}\right)$. By employing Zadeh's extension principle, we can compute the membership function for the priority vector $w \in Q^{n-1}$ in $D_{\lambda}$ as follows:

$$
\mu_{D_{\lambda}}(w)=\min _{i j}\left\{F_{i j}\left(w_{i} / w_{j}\right) \mid i, j=1, \ldots, n, i \neq j\right\}
$$

From the construction of membership function (7) and non-membership function (8), one may observe that they are extended in $(-\infty, \infty)$. That assumption ensures the non-emptiness of $D_{\lambda}$ for each $\lambda \in[0,1]$ over the simplex $Q^{n-1}$. In the following theorem, we demonstrate that fuzzy decision space $D_{\lambda}$ is a convex set.

Theorem 1. The fuzzy space $D_{\lambda}$ is convex.

Proof. Let us assume that $D_{\lambda}$ is a non-empty set. Consider the $\alpha$-cut set of the $D_{\lambda}$, which is denoted and defined as $D_{\lambda}(\alpha)=\left\{w \in Q^{n-1}: \mu_{D_{\lambda}}(w) \geqslant \alpha\right\}$. Take two points $w^{1}$ and $w^{2}$ such that $w^{1} \in D_{\lambda}(\alpha)$ and $w^{2} \in D_{\lambda}(\alpha)$. Then

$$
\mu_{D_{\lambda}}\left(w^{1}\right) \geqslant \alpha \text { and } \mu_{D_{\lambda}}\left(w^{2}\right) \geqslant \alpha
$$

It follows from Eq.(11) that for all $i, j=1,2, \ldots, n$ and $i \neq j$

$$
F_{i j}\left(w_{i}^{1} / w_{j}^{1}\right) \geqslant \alpha \text { and } F_{i j}\left(w_{i}^{2} / w_{j}^{2}\right) \geqslant \alpha
$$

Now, we consider a new point by taking the convex combination of $w^{1}$ and $w^{2}$, i.e., $w=\eta w^{1}+(1-$ $\eta) w^{2}$ where $\eta \in(0,1)$. One can easily note that Eq. (10) can be written as

$$
\begin{aligned}
& F_{i j}\left(w_{i} / w_{j}\right)= \mu_{i j}\left(w_{i} / w_{j}\right)+\lambda\left(1-\mu_{i j}\left(w_{i} / w_{j}\right)\right. \\
&\left.-v_{i j}\left(w_{i} / w_{j}\right)\right) \in[0,1] . \\
&=(1-\lambda) \mu_{i j}\left(w_{i} / w_{j}\right)+\lambda\left(1-v_{i j}\left(w_{i} / w_{j}\right)\right)
\end{aligned}
$$

Since both $\mu_{i j}($.$) and 1-v_{i j}($.$) are convex in na-$ ture, the membership function $F_{i j}($.$) (as in Eq. (12))$ being a convex combination of $\mu_{i j}($.$) and 1-v_{i j}($. 
is also convex for all $i, j=1, \ldots, n, i \neq j$. Now our intention is to show each of the ratios $w_{i} / w_{j}$ corresponding to the point $w$ can be expressed as a linear combination of ratios $w_{i}^{1} / w_{j}^{1}$ and $w_{i}^{2} / w_{j}^{2}$ corresponding to the points $w^{1}$ and $w^{2}$ respectively. It can be shown as follows:

$$
\begin{aligned}
w_{i} / w_{j}= & \frac{\eta w_{i}^{1}+(1-\eta) w_{i}^{2}}{\eta w_{j}^{1}+(1-\eta) w_{j}^{2}} \\
= & \frac{\eta}{\eta+(1-\eta) w_{j}^{2} / w_{j}^{1}} \frac{w_{i}^{1}}{w_{j}^{1}} \\
& \quad+\frac{1-\eta}{\eta w_{j}^{1} / w_{j}^{2}+(1-\eta)} \frac{w_{i}^{2}}{w_{j}^{2}} \\
= & \xi_{1} w_{i}^{1} / w_{j}^{1}+\xi_{2} w_{i}^{2} / w_{j}^{2}
\end{aligned}
$$

where $\xi_{1}=\frac{\eta}{\eta+(1-\eta) w_{j}^{2} / w_{j}^{1}}$ and $\xi_{2}=\frac{1-\eta}{\eta w_{j}^{1} / w_{j}^{2}+(1-\eta)}$. Clearly, $\xi_{1}, \xi_{2}>0$ and $\xi_{1}+\xi_{2}=1$.

As $F_{i j}($.$) is convex,$

$$
\begin{aligned}
F_{i j}\left(w_{i} / w_{j}\right) & =F_{i j}\left(\xi_{1} w_{i}^{1} / w_{j}^{1}+\xi_{2} w_{i}^{2} / w_{j}^{2}\right) \\
& \geqslant \min \left\{F_{i j}\left(w_{i}^{1} / w_{j}^{1}\right), F_{i j}\left(w_{i}^{2} / w_{j}^{2}\right)\right\} \geqslant \alpha
\end{aligned}
$$

for all $i, j=1,2, \ldots, n$ with $i \neq j$. It implies that,

$$
\mu_{D_{\lambda}}(w)=\min _{i j}\left\{F_{i j}\left(w_{i} / w_{j}\right) \mid i, j=1, \ldots, n, i \neq j\right\} \geqslant \alpha
$$

As $\alpha$ is arbitrary, the above inequality holds for all $\alpha \in(0,1)$. It follows that $w \in D_{\lambda}(\alpha)$. Hence, the fuzzy feasible region $D_{\lambda}$ is convex.

Convexity of $D_{\lambda}$ ensures that our prioritization method can always select a priority vector $w^{*} \in$ $Q^{n-1}$ which has highest satisfaction degree. Now, our aim is to find an optimal priority vector $w^{*} \in$ $Q^{n-1}$ such that it satisfies

$$
\alpha^{*}=\mu_{D_{\lambda}}\left(w^{*}\right)=\max _{w \in Q^{n-1}} \mu_{D_{\lambda}}(w) .
$$

For this purpose, we employ Bellman and Zadeh's principle ${ }^{24}$ of fuzzy decision making to solve the problem Eq. (13). Then, it transforms into the following crisp optimization problem:

$$
\begin{array}{ll}
\max & \alpha \\
\text { s.t. } & \left\{\begin{array}{l}
F_{i j}\left(w_{i} / w_{j}\right) \geqslant \alpha \\
\sum_{k=1}^{n} w_{k}=1, \\
w_{k}>0, k=1,2, \ldots, n \\
i, j=1, \ldots, n, i \neq j .
\end{array}\right.
\end{array}
$$

By changing the variation of indices $i$ and $j$, the optimization model (14) can also be written as

$$
\begin{array}{ll}
\max & \alpha \\
\text { s.t. } & \left\{\begin{array}{l}
F_{i j}\left(w_{i} / w_{j}\right) \geqslant \alpha, \\
F_{j i}\left(w_{j} / w_{i}\right) \geqslant \alpha, \\
\sum_{k=1}^{n} w_{k}=1 \\
w_{k}>0, k=1,2, \ldots, n, \\
i=1,2, \ldots, n-1, j=2, \ldots, n, j>i .
\end{array}\right.
\end{array}
$$

From the optimization model (15), it is clear that priority vector $w$ obtained by solving the model satisfies both the TIF ratio and its reciprocal simultaneously, which is essential to capture non-linearity and skewness of the reciprocal of GTIFNs ${ }^{16}$.

Using Eq. (12), optimization problem given in Eq. (15) can be put into the following form:

$$
\begin{array}{ll}
\max & \alpha \\
\text { s.t. } & \left\{\begin{array}{l}
(1-\lambda) \mu_{i j}\left(w_{i} / w_{j}\right)+\lambda\left(1-v_{i j}\left(w_{i} / w_{j}\right)\right) \geqslant \alpha, \\
(1-\lambda) \mu_{j i}\left(w_{j} / w_{i}\right)+\lambda\left(1-v_{j i}\left(w_{j} / w_{i}\right)\right) \geqslant \alpha, \\
\sum_{k=1}^{n} w_{k}=1, \\
w_{k}>0, k=1,2, \ldots, n, \\
i=1,2, \ldots, n-1, j=2, \ldots, n, j>i .
\end{array}\right.
\end{array}
$$

With the help of Eqs. (7) and (8), we transform optimization problem described in Eq. (16) into the following:

$$
\begin{aligned}
& \max \\
& \text { s.t. }\left\{\begin{array}{l}
w_{i}-u_{i j} w_{j}+\frac{\alpha}{t_{i j}}\left(u_{i j}-m_{i j}\right) w_{j} \leqslant 0 \\
-w_{i}+l_{i j}+\frac{\alpha}{t_{i j}}\left(m_{i j}-l_{i j}\right) w_{j} \leqslant 0 \\
w_{j}-\left(1 / l_{i j}\right) w_{i}+\frac{\alpha}{t_{i j}}\left(1 / l_{i j}-1 / m_{i j}\right) w_{i} \leqslant 0 \\
-w_{j}+\left(1 / u_{i j}\right)+\frac{\alpha}{t_{i j}}\left(1 / m_{i j}-1 / u_{i j}\right) w_{i} \leqslant 0 \\
\sum_{k=1}^{n} w_{k}=1 \\
w_{k}>0, k=1,2, \ldots, n, \\
i=1,2, \ldots, n, j=2, \ldots, n, j>i
\end{array}\right.
\end{aligned}
$$


where, $t_{i j}=(1-\lambda) \gamma_{i j}+\lambda\left(1-\delta_{i j}\right)$ for all $i=$ $1,2, \ldots, n-1, j=2, \ldots, n, j>i$.

When the value of the parameter $\lambda$ is given by the decision maker, the optimal solution $\left(\alpha^{*}, w^{*}\right)$ of the above non-linear problem can be obtained by using appropriate numerical optimization technique. In this paper, LINGO software is utilized to solve the non-linear model.

Remark 1. It may be noted that if there is no indeterminacy factor in the IFPP problem, i.e., $\gamma_{i j}=1$ for $i=1,2, \ldots, n-1, j=2, \ldots, n j>i$, then all the nonmembership functions $\left(v_{i j}().\right)$ are standard complements of the corresponding membership functions $\left(\mu_{i j}().\right)$. Therefore, there is no need of resolving indeterminacy factor and this fact can be considered into our model (17) by simply setting the value of $\lambda$ as zero. In that case, our model (17) turns into prioritization problem proposed by Rezaei et al. ${ }^{16}$ as follows:

$$
\begin{aligned}
& \max \alpha \\
& \text { s.t. }\left\{\begin{array}{l}
w_{i}-u_{i j} w_{j}+\alpha\left(u_{i j}-m_{i j}\right) w_{j} \leqslant 0 \\
-w_{i}+l_{i j}+\alpha\left(m_{i j}-l_{i j}\right) w_{j} \leqslant 0 \\
w_{j}-\left(1 / l_{i j}\right) w_{i}+\alpha\left(1 / l_{i j}-1 / m_{i j}\right) w_{i} \leqslant 0 \\
-w_{j}+\left(1 / u_{i j}\right)+\alpha\left(1 / m_{i j}-1 / u_{i j}\right) w_{i} \leqslant 0 \\
\sum_{k=1}^{n} w_{k}=1, \\
w_{k}>0, k=1,2, \ldots, n, \\
i=1,2, \ldots, n, j=2, \ldots, n, j>i .
\end{array}\right.
\end{aligned}
$$

Remark 2. Another important observation is that if we do not consider the satisfaction of reciprocal ratios apart from no indeterminacy factor, the IFPP model (17) reduces to the Mikhailov's model ${ }^{11}$ as follows:

$$
\begin{aligned}
& \max \alpha \\
& \text { s.t. }\left\{\begin{array}{l}
w_{i}-u_{i j} w_{j}+\alpha\left(u_{i j}-m_{i j}\right) w_{j} \leqslant 0, \\
-w_{i}+l_{i j}+\alpha\left(m_{i j}-l_{i j}\right) w_{j} \leqslant 0, \\
\sum_{k=1}^{n} w_{k}=1, \\
w_{k}>0, k=1,2, \ldots, n, \\
i=1,2, \ldots, n, j=2, \ldots, n, j>i .
\end{array}\right.
\end{aligned}
$$

Based on the solution of our optimization model (16), there are some important observations, which are described below.

Note 1 For a fixed value of $\lambda$, the positive value of $\alpha^{*}$ indicates that the initial intuitionistic fuzzy judgments satisfy the inequalities $l_{i j}<w_{i}^{*} / w_{j}^{*}<u_{i j}$ and $1 / u_{i j}<w_{j}^{*} / w_{i}^{*}<1 / l_{i j}$ for all $i=1,2, \ldots, n, j=$ $2, \ldots, n, j \neq i$. This implies that there is reasonable consistency in the initial generalized intuitionistic fuzzy comparison matrix. On the other hand, the negative value of $\alpha^{*}$ suggests that the priority vector $w^{*}$ does not satisfy the inequalities $l_{i j}<w_{i}^{*} / w_{j}^{*}<u_{i j}$ and $1 / u_{i j}<w_{j}^{*} / w_{i}^{*}<1 / l_{i j}$ for at least one pair of values of $(i, j)$ where $i=1,2, \ldots, n, j=2, \ldots, n, j \neq i$. Therefore, the negative value of $\alpha^{*}$ means that there is a strong inconsistency among the initial generalized intuitionistic fuzzy pair-wise comparison matrix. In fact, the optimal value of $\alpha$ describes the combined level of satisfaction of intuitionistic fuzzy inequities (5) with some indeterminacy factor. Owing to this attractive feature of $\alpha^{*}$, some researchers 11,16 have interpreted $\alpha^{*}$ as a consistency index of generalized intuitionistic fuzzy pair-wise comparison matrix.

Note 2 There is an interesting feature of $\alpha^{*}$. As the negative level of $\alpha^{*}$ indicates a strong inconsistency in initial generalized intuitionistic fuzzy pairwise comparison matrix, decision maker may not be satisfied with derived priority vector. In such case, the decision maker may think about re-evaluating initial comparison matrix to improve satisfaction level.

Note 3 The parameter $\lambda$ also can be interpreted in another way. The membership function of ratio $w_{i} / w_{j}$, can written as $F\left(w_{i} / w_{j}\right)=(1-$ ג) $\mu_{i j}\left(w_{i} / w_{j}\right)+\lambda\left(1-v_{i j}\left(w_{i} / w_{j}\right)\right)$

The preceding equation may be viewed as a linear combination of upper and lower membership function of interval valued fuzzy numbers $\left[\left(l_{i j}, m_{i j}, u_{i j} ; \gamma_{i j}\right),\left(l_{i j}, m_{i j}, u_{i j} ; 1-\delta_{i j}\right)\right]$ (see Fig. 1). According to Hurwicz pessimism-optimism criterion ${ }^{25}$, one may interpret the value of $\lambda(0 \leqslant \lambda \leqslant 1)$ as a decision maker's degree of optimism. The views of the decision maker can be explained as follows:

- when $0.5<\lambda \leqslant 1$, decision maker takes optimistic stand (Fig. 1 (c)) 
Table 2: Generalized intuitionistic fuzzy pair-wise comparison matrix

\begin{tabular}{cccc}
\hline & $A_{1}$ & $A_{2}$ & $A_{3}$ \\
\hline$A_{1}$ & $([1,1,1] ; 1,0)$ & $([1 / 3.5,1 / 3,1 / 2.5] ; 0.8,0.1)$ & $([1.5,2,2.5] ; 0.8,0.1)$ \\
$A_{2}$ & $([2.5,3,3.5] ; 0.8,0.1)$ & $([1,1,1] ; 1,0)$ & $([2.5,3,3.5] ; 0.8,0.1)$ \\
$A_{3}$ & $([1 / 2.5,1 / 2,1 / 1.5] ; 0.8,0.1)$ & $([2.5,3,3.5] ; 1,0)$ & $([1,1,1] ; 1,0)$
\end{tabular}

- when $0 \leqslant \lambda<0.50$, the decision maker is pessimistic (Fig.1 (a))

- when $\lambda=0.5$, the decision maker is neutral (Fig. $1(b))$

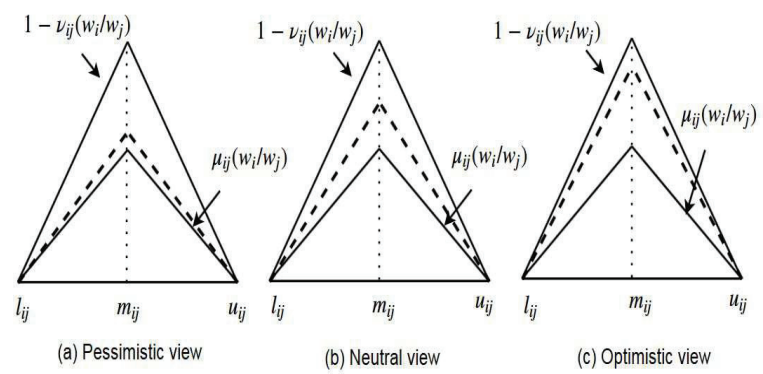

Fig. 1. Decision makers different view points.

With the changes of decision maker's view point, according to $\lambda$, we may obtain different priority vectors with varying satisfaction level. Based on the decision maker's knowledge of the studied system and expertise, one can select the suitable value of the parameter $\lambda$.

\section{Numerical Example}

In this section, four examples are examined by the proposed method. First example is taken from the paper of Sadiq and Tesfamariam ${ }^{15}$ to show that the priority weights, generated by the geometric mean approach, deviate significantly from decision maker's initial judgments. It is also shown that the proposed method overcomes the drawback. Second example is used to demonstrate the effect of the parameter $\lambda$, which describes decision maker's attitude of resolving hesitancy, on priority weights. In the third example, we show that logarithmic preference programming proposed by Wang and Chin ${ }^{26}$ may produce unreasonable result in the case of consistent pair-wise comparison matrices and we also compare the results with the proposed approach. In the last example, we provide a real application of the proposed method in the supplier location selection problem.

Example 1. Consider the following three dimensional prioritization problem, described in Table 2. First we will apply the intuitionistic fuzzy weight generation process proposed by Sadiq and Tesfamarian ${ }^{15}$ on the above problem. Finding intuitionistic fuzzy weights of the prioritization problem means to generate three IFNs $\widetilde{w}_{1}, \widetilde{w}_{2}, \widetilde{w}_{3}$ such that they satisfy the conditions $\widetilde{w}_{1}+\widetilde{w}_{2}+\widetilde{w}_{3}=\widetilde{1}$ and $\widetilde{a}_{i j}=\widetilde{w}_{i} / \widetilde{w}_{j}$ for $i, j=1,2,3$. By taking geometric mean approach and applying normalization procedure as proposed by ${ }^{15}$, we obtain intuitionistic fuzzy weights as follows: $\quad \widetilde{w}_{1}=([0.15,0.25,0.41] ; 0.8,0.1)$, $\widetilde{w}_{2}=([0.39,0.59,0.76] ; 0.8,0.1), \widetilde{w}_{3}=([0.08,0.16$, $0.38] ; 0.8,0.1)$

From the above obtained weights, the resulting intuitionistic fuzzy ratio, corresponding to the pairwise comparison $\widetilde{a}_{23}$, can be calculated by using intuitionistic fuzzy division operator as $\widetilde{w}_{2} / \widetilde{w}_{3}=$ $([1.33,3.69,8.44] ; 0.8,0.1)$.

One may observe that the derived intuitionistic fuzzy ratio $([1.33,3.69,8.44] ; 0.8,0.1)$ is strongly skewed, in contrast to the given initial judgment $([2.5,3,3.5] ; 0.8,0.1)$, provided in Table 2. Hence, we may argue that geometric mean approach may alter the initial imprecise preferences due to using approximate fuzzy multiplication and division operators of GTIFNs.

On the other hand, for finding the priority weights of the factors $A_{1}, A_{2}$ and $A_{3}$, the proposed model (Eq. 17) leads us to solve the following opti- 
Table 3: Generalized intuitionistic fuzzy pair-wise comparison matrix

\begin{tabular}{cccc}
\hline & Pricing & Service quality & Delivery time \\
\hline Pricing & 1 & $([2,3,4], 0.8,0.1)$ & $([1,2,3], 0.8,0.1)$ \\
Service quality & $([1 / 4,1 / 3,1 / 2], 0.8,0.1)$ & 1 & $([1 / 3,1 / 2,1], 0.8,0.1)$ \\
Delivery time & $([1 / 3,1 / 2,1], 0.8,0.1)$ & $([1,2,3], 0.8,0.1)$ & 1
\end{tabular}

mization problem

$$
\begin{aligned}
& \max \alpha \\
& \text { s.t. }\left\{\begin{array}{l}
w_{1}-\frac{w_{2}}{2.5}+\frac{\alpha}{((1-\lambda) 0.8+0.9 \lambda) 15} w_{2} \leqslant 0 \\
-w_{1}+\frac{w_{2}}{3.5}+\frac{\alpha}{((1-\lambda) 0.8+0.9 \lambda) 21} w_{2} \leqslant 0 \\
w_{1}-2.5 w_{3}+\frac{\alpha}{((1-\lambda) 0.8+0.9 \lambda) 0.5} w_{3} \leqslant 0 \\
-w_{1}+1.5 w_{3}+\frac{\alpha}{((1-\lambda) 0.8+0.9 \lambda) 0.5} w_{3} \leqslant 0 \\
w_{2}-3.5 w_{3}+\frac{\alpha}{((1-\lambda) 0.8+0.9 \lambda) 0.5} w_{3} \leqslant 0 \\
-w_{2}+2.5 w_{3}+\frac{\alpha}{((1-\lambda) 0.8+0.9 \lambda) 0.5} w_{3} \leqslant 0 \\
w_{3}-\frac{w_{1}}{1.5}+\frac{\alpha}{((1-\lambda) 0.8+0.9 \lambda) 6} w_{1} \leqslant 0 \\
-w_{3}+\frac{w_{2}}{2.5}+\frac{\alpha}{((1-\lambda) 0.8+0.9 \lambda) 10} w_{1} \leqslant 0 \\
w_{3}-\frac{w_{2}}{2.5}+\frac{\alpha}{((1-\lambda) 0.8+0.9 \lambda) 15} w_{2} \leqslant 0 \\
-w_{3}+\frac{w_{2}}{3.5}+\frac{\alpha}{((1-\lambda) 0.8+0.9 \lambda) 21} w_{2} \leqslant 0 \\
w_{1}+w_{2}+w_{3}=1, \\
w_{1}, w_{2}, w_{3} \geqslant 0 .
\end{array}\right.
\end{aligned}
$$

Setting the indeterminacy resolving parameter $\lambda=$ 0.5 (i.e., decision maker takes neutral stand), we obtain the optimal solution of the above optimization model as follows: $\alpha^{*}=-0.52$ and $w_{1}^{*}=0.2477$, $w_{2}^{*}=0.5619, w_{3}^{*}=0.1904$. The negative value of $\alpha^{*}$ indicates that there is inconsistency in judgments. However, the ratio $w_{2}^{*} / w_{3}^{*}=2.9512$ belongs to the initial support of $\widetilde{a}_{23}$. The negative value of $\alpha^{*}$ is due to the little violation of the constraints $l_{12}<w_{1} / w_{2}<u_{12}$ and $l_{31}<w_{3} / w_{1}<u_{31}$.

Example 2. Consider another example of a prioritization problem of three main attributes: pricing, service quality and delivery time with respect to a goal for evaluating services. The intuitionistic fuzzy pair-wise comparison matrix is presented in Table 3.

For this comparison matrix, model (17) can be written as

$$
\begin{aligned}
& \max \alpha \\
& \text { s.t. }\left\{\begin{array}{l}
w_{1}-3 w_{3}+\frac{\alpha}{(1-\lambda) 0.8+0.9 \lambda} w_{3} \leqslant 0, \\
-w_{1}+w_{3}+\frac{\alpha}{(1-\lambda) 0.8+0.9 \lambda} w_{3} \leqslant 0, \\
w_{2}-w_{3}+\frac{\alpha}{((1-\lambda) 0.8+0.9 \lambda) 6} w_{3} \leqslant 0, \\
-w_{2}+\frac{w_{3}}{3}+\frac{\alpha}{((1-\lambda) 0.8+0.9 \lambda) 2} w_{3} \leqslant 0, \\
-w_{2}+\frac{w_{1}}{4}+\frac{\alpha}{((1-\lambda) 0.8+0.9 \lambda) 12} w_{1} \leqslant 0, \\
w_{2}-\frac{w_{1}}{2}+\frac{\alpha}{((1-\lambda) 0.8+0.9 \lambda) 6} w_{1} \leqslant 0, \\
-w_{3}+\frac{w_{1}}{3}+\frac{\alpha}{((1-\lambda) 0.8+0.9 \lambda) 6} w_{1} \leqslant 0, \\
w_{3}-w_{1}+\frac{\alpha}{((1-\lambda) 0.8+0.9 \lambda) 2} w_{1} \leqslant 0, \\
-w_{3}+w_{2}+\frac{\alpha}{((1-\lambda) 0.8+0.9 \lambda)} w_{2} \leqslant 0, \\
w_{3}-3 w_{2}+\frac{\alpha}{((1-\lambda) 0.8+0.9 \lambda)} w_{2} \leqslant 0, \\
w_{1}+w_{2}+w_{3}=1, \\
w_{1}, w_{2}, w_{3}>0 .
\end{array}\right.
\end{aligned}
$$

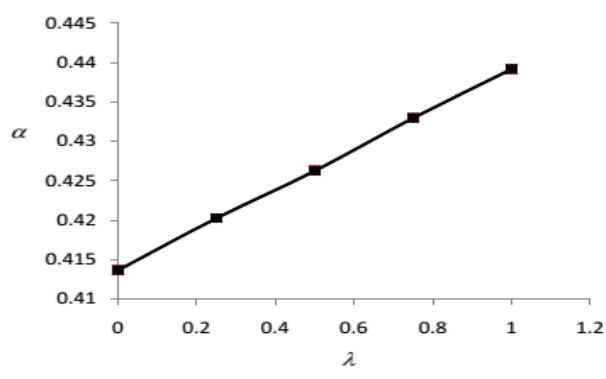

Fig. 2. Changes in priority vector and satisfaction level with respect to parameter $\lambda$.

Taking neutral approach for resolving hesitancy, i.e., $\lambda=0.5$, we get the optimal solution for $w^{*}$ and $\alpha^{*}$ as follows: $w_{1}^{*}=0.5583, w_{2}^{*}=0.1629$, $w_{3}^{*}=0.2788, \alpha^{*}=0.4268$. The positive value of $\alpha^{*}$ indicates there is reasonable consistency in pairwise judgments and all the ratios are within their fuzzy supports. If the decision maker takes different 
stands to resolve hesitancy by varying the parameter $\lambda$, we obtain new priority vectors with different satisfaction degrees. Table 4 describes the changes in $w^{*}$ with the changes in $\lambda$, while Figure 2 depicts changes in $\alpha^{*}$ with the change in $\lambda$.

Table 4. Changes in priority vector and satisfaction with respect to parameter $\lambda$

\begin{tabular}{cc}
\hline$\lambda$ & $w^{*}$ \\
\hline 0 & $(0.5592,0.1639,0.2769)$ \\
$1 / 4$ & $(0.5587,0.1634,0.2779)$ \\
$1 / 2$ & $(0.5583,0.1629,0.2788)$ \\
$3 / 4$ & $(0.5578,0.1625,0.2797$ \\
1 & $(0.5574,0.1620,0.2806)$ \\
\hline
\end{tabular}

Example 3. Consider a $3 \times 3$ prioritization matrix $\widetilde{A}=\left(\widetilde{a}_{i j}\right)_{3 \times 3}$ with the entries $\widetilde{a}_{12}=\langle(1,2,3) ; 1,0\rangle$, $\widetilde{a}_{13}=\langle(2,4,6) ; 1,0\rangle$ and $\widetilde{a}_{23}=\langle(2,2,2) ; 1,0\rangle$. Clearly, $\widetilde{A}$ can be represented as an IF fuzzy pairwise comparison matrix. Also, it is consistent according to the definition of consistency proposed by Laarhoven and Pedrycz ${ }^{3}$ (the consistency is realized by treating the given data, i.e., pair-wise comparison ratios as fuzzy numbers since the corresponding non-membership degrees are zero). Now, we employ fuzzy logarithmic preference programming proposed by Wang and Chin's ${ }^{26}$ to derive crisp priority vector $w=\left(w_{1}, w_{2}, w_{3}\right)$. According to them, the optimization model for this fuzzy pair-wise comparison matrix is as

$$
\begin{aligned}
& \max (1-\alpha)^{2}+M \cdot \sum_{i=1}^{2} \sum_{j=i+1}^{3} \eta_{i j}^{2}+\chi_{i j}^{2} \\
& \text { s.t. }\left\{\begin{array}{l}
x_{1}-x_{2}-\alpha \ln (2 / 1)+\eta_{12}^{2} \geqslant \ln (1), \\
-x_{1}+x_{2}-\alpha \ln (3 / 2)+\chi_{12}^{2} \geqslant-\ln (3), \\
x_{1}-x_{3}-\alpha \ln (4 / 2)+\eta_{13}^{2} \geqslant \ln (2), \\
-x_{1}+x_{3}-\alpha \ln (6 / 4)+\chi_{13}^{2} \geqslant-\ln (6), \\
x_{2}-x_{3}-\alpha \ln (2 / 2)+\eta_{23}^{2} \geqslant \ln (2), \\
-x_{2}+x_{3}-\alpha \ln (2 / 2)+\chi_{23}^{2} \geqslant-\ln (2), \\
\alpha, x_{1}, x_{2}, x_{3}, \eta_{12} . \eta_{13}, \eta_{23}, \chi_{12}, \chi_{13}, \chi_{23} \geqslant 0
\end{array}\right.
\end{aligned}
$$

Taking sufficiently large value of $M$, i.e., $M=1000$, we obtain the optimal solution as follows:

$\alpha=0.397, \quad x_{1}=1.23, x_{2}=0.286, x_{3}=$ 0.286, $\eta_{12}=0, \eta_{13}=0, \eta_{23}=0, \chi_{12}=0, \chi_{13}=$
$0, \chi_{23}=0$.

Then by utilizing $w_{i}=\frac{\exp \left(x_{i}\right)}{\sum_{i=1}^{3} \exp \left(x_{i}\right)}$, we get the priority vector as follows: $w_{1}=0.56359 w_{2}=0.218, w_{3}=$ 0.218

It may be noted that instead of consistency in initial fuzzy pair-wise comparison matrix, the priority vector derived from Wang and Chin model is quite unreasonable. As $\widetilde{a}_{23}=w_{2} / w_{3}=1$, the priority weight does not preserve the initial preference of the decision maker. On the other hand, by our proposed model (17) for this same IF fuzzy pair-wise comparison matrix generates the priority weights as follows: $w_{1}=0.60, w_{1}=0.26, w_{3}=0.13$. The priority vector is quite consistent with the decision maker's initial preferences. In case of the entry $\widetilde{a}_{23}$, we have $\widetilde{a}_{23}=w_{2} / w_{3}=2$, which completely agrees with the decision maker's initial preference. Thus, the proposed approach can overcome the demerit of Wang and Chin's approach.

In the next example, we apply the prioritization method in MADM problem concerning site selection of motor company.

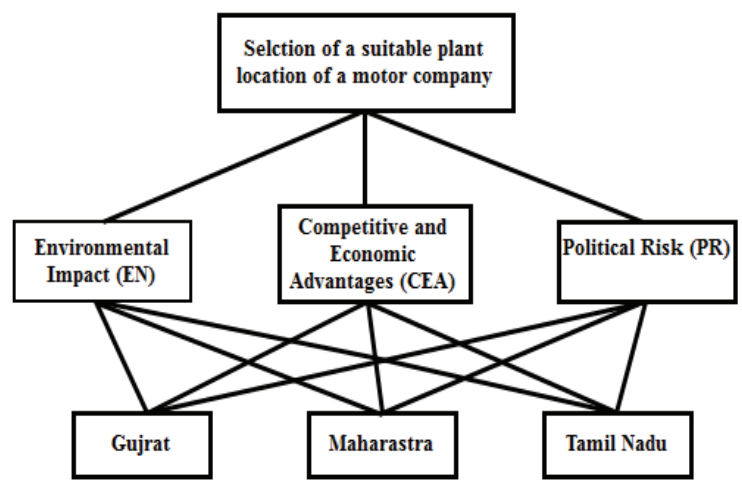

Fig. 3. Decision hierarchy

Example 4. An Indian motor company, $\mathrm{ABC}$, is planning to invest in a new manufacturing plant. The aim of the company is to select the most suitable location among the alternatives. The three criteria taken into consideration are the environmental norm (EN), competitive and economic advantages (CEA) and political risk (PR). The locations under consideration, treated as alternatives, are Gujrat, Maharastra and Tamil Nadu. The main goal of the decision maker is to select an alternative which satisfies all 
Table 5: Generalized intuitionistic fuzzy pair-wise comparison matrix of three main attributes with respect to goal and its priority

\begin{tabular}{cccccc}
\hline & EN & CEA & PR & IFPP Priority & $\alpha^{*}$ \\
\hline EN & $([1,1,1] ; 1,0)$ & $([2 / 5,1 / 2,2 / 3] ; 0.8,0.1)$ & $([1,3 / 2,2] ; 0.8,0.1)$ & 0.27 & \\
CEA & $([3 / 2,2,5 / 2], 0.8,0.1)$ & $([1,1,1] ; 1,0)$ & $([5 / 2,3,7 / 2] ; 0.8,0.1)$ & 0.55 & 0.800 \\
PR & $([1 / 2,2 / 3,1] ; 0.8,0.1)$ & $([2 / 7,1 / 3,2 / 5], 0.8,0.1)$ & $([1,1,1] ; 1,0)$ & 0.18 &
\end{tabular}

Table 6: Generalized intuitionistic fuzzy pair-wise comparison matrix with respect to EN

\begin{tabular}{cccccr}
\hline & Gujrat & Maharastra & Tamil Nadu & IFPP Priority & $\alpha^{*}$ \\
\hline Gujrat & $([1,1,1] ; 1,0)$ & $([3 / 2,2,5 / 2] ; 0.8,0.1)$ & $([1 / 2,2 / 3,1] ; 0.8,0.1)$ & 0.37 & \\
Maharastra & $([2 / 5,1 / 2,2 / 3], 0.8,0.1)$ & $([1,1,1] ; 1,0)$ & $([1 / 2,1,3 / 2] ; 0.8,0.1)$ & 0.23 & 0.141 \\
Tamil Nadu & $([1,3 / 2,2] ; 0.8,0.1)$ & $([2 / 3,1,2] ; 0.8,0.1)$ & $([1,1,1] ; 1,0)$ & 0.40 &
\end{tabular}

Table 7: Generalized intuitionistic fuzzy pair-wise comparison matrix with respect to CEA

\begin{tabular}{cccccc}
\hline & Gujrat & Maharastra & Tamil Nadu & IFPP Priority & $\alpha^{*}$ \\
\hline Gujrat & $([1,1,1] ; 1,0)$ & $([5 / 2,3,7 / 2] ; 0.8,0.1)$ & $([3 / 2,2,5 / 2] ; 0.8,0.1)$ & 0.55 & \\
Maharastra & $([2 / 7,1 / 3,2 / 5], 0.8,0.1)$ & $([1,1,1] ; 1,0)$ & $([1 / 2,1,3 / 2] ; 0.8,0.1)$ & 0.20 & 0.485 \\
Tamil Nadu & $([2 / 5,1 / 2,2 / 3] ; 0.8,0.1)$ & $([2 / 3,1,2] ; 0.8,0.1)$ & $([1,1,1] ; 1,0)$ & 0.25 &
\end{tabular}

Table 8: Generalized intuitionistic fuzzy pair-wise comparison matrix with respect to PR

\begin{tabular}{cccccc}
\hline & Gujrat & Maharastra & Tamil Nadu & IFPP Priority & $\alpha^{*}$ \\
\hline Gujrat & $([1,1,1] ; 1,0)$ & $([2 / 5,1 / 2,2 / 3] ; 0.8,0.1)$ & $([1 / 2,2 / 3,1] ; 0.8,0.1)$ & 0.22 & 0.205 \\
Maharastra & $([3 / 2,2,5 / 2], 0.8,0.1)$ & $([1,1,1] ; 1,0)$ & $([1 / 2,1,3 / 2] ; 0.8,0.1)$ & 0.36 & \\
Tamil Nadu & $([1,3 / 2,2] ; 0.8,0.1)$ & $([2 / 3,1,2] ; 0.8,0.1)$ & $([1,1,1] ; 1,0)$ & 0.42 &
\end{tabular}

the attributes in the best way.

We solve the decision making problem by applying our proposed ituitionistic fuzzy AHP method based on IFPP. The first step in applying the IF AHP is to construct a hierarchy structure of the decision problem. The hierarchy of the goal, criteria and alternatives are shown in Figure 3.

The next step is to construct a pair-wise comparison matrix. In real-world complex system, pair-wise comparison of decision elements involves fuzzy and qualitative aspects, and measuring such aspects requires decision makers' perceptions which contain subjectivity, imprecision and vagueness. Therefore, it is difficult for the decision makers to provide their preferences against the alternatives by using exact numerical values. A decision maker might feel more comfortable to articulate their preferences using words by means of linguistic terms. The uncertainty and hesitation involved in subjective judgments are expressed via linguistic terms which are suitably quantified by GTIFNs.

Decision maker first constructs the pair-wise comparison matrix of criteria, as shown in Table 5. From the pair-wise comparison matrix, the weights of the criteria are computed by employing our proposed IFPP model and are presented in the fifth column of Table 5 with the corresponding value of $\alpha^{*}$ in the last column of that table. It is noted here that for resolving hesitancy corresponding to each of the comparison matrices, we have taken neutral approach, i.e., hesitancy parameter $\lambda$ is set as 0.5 .

With respect to each criteria, alternatives are 
compared and pair-wise comparison matrices are formed, as shown in Tables 6-8. The priority weights of the alternatives with respect to each attribute are computed by utilizing IFPP model (17) and shown in the fifth column of the Tables 6-8 with the corresponding value of $\alpha^{*}$ in the last column.

Finally the priority weight of each location is obtained by weights per location multiplied by weights of the corresponding criterion. The highest score of the location gives the idea about the best global suitable location for investing new manufacturing plant. The results are shown in Table 9. According to the final score Gujrat is the most suitable location and Tamil Nadu is the alternative location after this.

Table 9. Priority weight of the alternatives with respect to goal

\begin{tabular}{|c|c|c|c|c|}
\hline & EN & CEA & PR & $\begin{array}{c}\text { Final IFPP } \\
\text { Priority }\end{array}$ \\
\hline Weights of attributes $\rightarrow$ & 0.27 & 0.55 & 0.18 & \\
\hline $\begin{array}{l}\text { Alternatives' weight } \\
\text { with respect to attribute } \\
\quad \downarrow\end{array}$ & & & & \\
\hline Gujrat & 0.37 & 0.55 & 0.22 & 0.44 \\
\hline Maharstra & 0.23 & 0.20 & 0.36 & 0.24 \\
\hline Tamil Nadu & 0.40 & 0.25 & 0.42 & 0.32 \\
\hline
\end{tabular}

\section{Conclusion}

In this study, we have proposed preference programming based weight determination method of intuitionistic fuzzy AHP, where decision maker expresses his/her judgments of pair-wise comparisons by using GTIFNs. As, IFNs can describe the uncertainty and vagueness of subjective judgments in a more comprehensive way than its' counterpart fuzzy numbers, the use of it in AHP allows us to more accurately describe the decision making process. We have examined several examples to show that the proposed method is capable to compute priority weights more accurately while other existing approaches fail to provide reasonable results. The main advantages of the proposed method are pointed out as follows:

- in the proposed method for deriving the prior- ity weights additional aggregation, normalization and ranking procedures of IFNs are not required, which sometimes lead us to obtain unreliable final priorities

- the parameter $\lambda$ associated with value function $F_{i j}$ gives the flexibility to the decision makers for solving their hesitation at different levels

- the optimal value of the parameter $\alpha^{*}$ can be used for measuring consistency of the initial set of IF judgments.

- this method is very straightforward and simple in computation.

- in the present context, for easy understanding and obvious computational advantage, the functions $\mu_{i j}$ and $v_{i j}$, used in describing the membership and non-membership functions are taken as linear functions. However, depending on the practical situations, these functions can be non-linear in nature.

\section{Acknowledgments}

The first author gratefully acknowledges the financial support provided by the Council of Scientific and Industrial Research, New Delhi, India (Award No. 09/1023(007)/2011-EMR-I).

\section{References}

1. T. L. Saaty, "A scaling method for priorities in hierarchical structures," J. Math. Psychol., 15, 234-281 (1977).

2. T. L. Saaty, "An exposition of the AHP in reply to the paper "remarks on the analytic hierarchy process"," Management Sci., 36, 259-268 (1990).

3. P. Van Laarhoven and W. Pedrycz, "A fuzzy extension of Saaty's priority theory," Fuzzy Sets Syst., 11, 199227 (1983).

4. M. Wagenknecht and K. Hartmann, "On fuzzy rankordering in polyoptimization," Fuzzy Sets Syst., 11, 243-251 (1983).

5. J. Buckley, "Fuzzy hierarchical analysis," Fuzzy Sets Syst., 17, 233-247 (1985).

6. D. -Y. Chang, "Applications of the extent analysis method on fuzzy AHP," Eur. J. Oper. Res., 95, 649655, (1996). 
7. C. H. Cheng and D. -L. Mon, "Evaluating weapon system by Analytical Hierarchy Process based on fuzzy scales," Fuzzy Sets Syst., 63, 1-10 (1994).

8. R. Xu, "Fuzzy least-squares priority method in the analytic hierarchy process," Fuzzy Sets Syst., 112, 395404 (2000).

9. R. Csutora and J. J. Buckley, "Fuzzy hierarchical analysis: the Lambda-Max method," Fuzzy Sets Syst., 120, 181-195 (2001).

10. L. Mikhailov, "Deriving priorities from fuzzy pairwise comparison judgments," Fuzzy Sets Syst., 134, 365-385 (2003).

11. L. Mikhailov and P. Tsvetinov, "Evaluation of services using a fuzzy analytic hierarchy process," Appl. Soft Comput., 5, 23-33 (2004).

12. Y. -M. Wang and K. -S. Chin, "An eigenvector method for generating normalized interval and fuzzy weights," Appl. Math. Comput., 181, 1257-1275 (2006).

13. Y. -M. Wang and K. -S. Chin, "A linear goal programming priority method for fuzzy analytic hierarchy process and its applications in new product screening," Int. J. Approx. Reason., 49, 451-465 (2008).

14. K. T. Atanassov, "Intuitionistic fuzzy sets," Fuzzy Sets Syst., 20, 87-96 (1986).

15. R. Sadiq and S. Tesfamariam, "Environmental decision-making under uncertainty using intuitionistic fuzzy analytic hierarchy process (IF-AHP)," Stoch. Environ. Res. Risk Assess., 23, 75-91 (2007).

16. J. Rezaei, R. Ortt, and V. Scholten, "An improved fuzzy preference programming to evaluate entrepreneurship orientation," Appl. Soft Comput., 13, 2749-2758 (2013).

17. D. -F. Li, "A ratio ranking method of triangular intu- itionistic fuzzy numbers and its application to MADM problems," Comput. Math. with Appl., 60, 1557-1570 (2010).

18. B. Dutta and D. Guha, "Trapezoidal intuitionistic fuzzy Bonferroni means and its application in multiattribute decision making," Proc. IEEE Int. Conf. Fuzzy Syst., 1-8 (2008).

19. D. Guha and D. Chakroborty, "A theoretrical development of distance measure for intuitionistic fuzzy numbers," International Journal of Mathematics and Mathematical Sciences, Article ID 949143 (2010).

20. S. -H. Chen, "Operations of fuzzy numbers with step form membership function using function principle," Inf. Sci., 108, 149-155 (1998).

21. P. P. Angelov, "Optimization in an intuitionistic fuzzy environment," Fuzzy Sets Syst., 86, 299-306, 1997.

22. R. R. Yager, "Some aspects of intuitionistic fuzzy sets," Fuzzy Optim. Decis. Mak., 8, 67-90 (2009).

23. D. Dubey, S. Chandra, and A. Mehra, "Fuzzy linear programming under interval uncertainty based on IFS representation," Fuzzy Sets Syst., 188, 68-87 (2012).

24. R. E. Bellman, L. A. Zadeh, "Decision-making in a fuzzy environment," Manage. Sci., 17, B141-B164 (1970).

25. L. Hurwicz, "The generalized bayes minimax principle: a criteria for decision making under uncertainty," Cowles Commision, Discussion Paper Statistics, 335 (1951).

26. Y. -M. Wang and K. -S. Chin, "Fuzzy analytic hierarchy process: A logarithmic fuzzy preference programming methodology," Int. J. Approx. Reason., 52, 541$553(2011)$. 